\title{
Effect of Soil Physico-Chemical Properties and Plant Species on Bacterial Diversity in Semi-Arid Parts in Central Sudan. Part III: AL-Gaeli Region, Khartoun North
}

\author{
Hatil Hashim EL-Kamali1 ${ }^{*}$, Hayat Ibrahim Hassan', Mustafa Siddig Mohamed EL-Kheir² \\ ${ }^{1}$ Department of Botany, Faculty of Science and Technology, Omdurman Islamic University, Omdurman, Sudan \\ ${ }^{2}$ Department of Biotechnology, Faculty of Science and Technology, Omdurman Islamic University, Omdurman, Sudan \\ Email: hatilhashim@gmail.com
}

How to cite this paper: EL-Kamali, H.H., Hassan, H.I. and EL-Kheir, M.S.M. (2017) Effect of Soil Physico-Chemical Properties and Plant Species on Bacterial Diversity in Semi-Arid Parts in Central Sudan. Part III: AL-Gaeli Region, Khartoun North. Open Access Library Journal, 4: e3337.

https://doi.org/10.4236/oalib.1103337

Received: December 27, 2016

Accepted: February 27, 2017

Published: March 2, 2017

Copyright $\odot 2017$ by authors and Open Access Library Inc.

This work is licensed under the Creative Commons Attribution International License (CC BY 4.0).

http://creativecommons.org/licenses/by/4.0/

\begin{abstract}
Bacterial diversity and total viable counts of bacteria of the different soil samples from three different localities in AL-Gaeli region, Khartoum North: Kunger, Gary and WadiAb-Gadad sub-regions were carried out. Soil physical and chemical characteristics ( $\mathrm{pH}, \mathrm{EC}, \mathrm{SP}$, soluble cations: $\mathrm{Na}, \mathrm{K}, \mathrm{Ca}, \mathrm{Mg}$ and anion $\mathrm{P}$, organic carbon, total nitrogen and soil texture) in each studied sub-regions were measured. Qualitative analysis of microorganisms isolated from the studied soil samples reveal a total of eight different species of bacteria, of which two are unidentified. The six species are classified under Bacillus genera. In study region soil samples, total bacterial counts ranged from $8.5 \times$ $10^{5} \mathrm{cfu} \cdot \mathrm{g}^{-1}$ to $1 \times 10^{4}$. The quantitative data on microbial population recorded in the present study was analysed using two diversity indices. Actinomyces spp. and Streptomyces spp. were the most abundant microorganisms identified in the three sub-regions. There were obvious differences in correlation coefficients among the selected criteria that $65 \%$ from the total number of correlation coefficients were positively correlated between bacterial counts and soil physico-chemical properties. The development of molecular techniques of microbial identification, coupled with traditional methods is promising areas for continued research.
\end{abstract}

\section{Subject Areas}

Ecology

\section{Keywords}

Microbial Diversity, Physico-Chemical Properties, Soils, Semi-Arid Region, Khartoum North, Central Sudan 


\section{Introduction}

Continuing our research works on the relationships between soil physicochemical properties, plant species and soil microorganisms populations in semi-arid parts in Sudan [1] [2], we have study correlations between these parameters in AL-Gaeli region, Khartoum North, Central Sudan.

Soil is a complex habitat, inhabited by a large number of different organisms. Among these, bacteria and fungi are the most important since they are responsible for the vast bulk of decomposition, and also make up the largest part of the biomass in soil. Many of the essential transformations in the nitrogen, sulphur, phosphorus and other element cycles are mediated by microbes. Bacteria are the most abundant microorganism group in soil and can attain concentrations of more than $10^{8}$ cells per gram of soil, or $10^{11}$ per gram organic material (cited by Pettersson, 2004) [3].

Soil organisms (biota) carry out a wide range of processes that are important for soil health and fertility in both natural and manged agricultural soils. The total number of organisms, the diversity of species and the activity of the soil biota will fluctuate as the soil environment changes. These changes may be caused by natural or imposed systems. The activity of soil organisms can be divided into four functions: 1) regulation of organic matter turnover and nutrient cycling, 2) biological degradation, 3) maintenance of soil structure, and 4) interaction with plants. The main factors contributing to the soil environment are: i) soil texture and structure; ii) nutrient status; iii) soil $\mathrm{pH}$; iv) moisture and temperature; v) surface plants; vi) Inputs and vii) compaction. There are a number of environmental factors that affect the bacterial community. Some of these factors are called modulators, in contrast that resources that the microbial community needs for growth (e.g. carbon, nitrogen). The difference between modulators and resources is that organisms actively compete for resources, while they cannot compete for modulators. As one of the most important environmental factors, $\mathrm{pH}$ has a determining role in the type of organisms that predominate in different soils (cited by Pettersson, 2004) [3].

Soil microbial communities develop in response to constraints, and selection pressures in their environmental (physical, chemical and biological). The chemical and biological constraints have been studied extensively [4] [5] [6]. In contrast, ways in which the physical environment of soil exerts control over community structure and diversity are more poorly understood.

The objectives of this study were: 1) to obtain a better understanding of the correlations between microbial population and physico-chemical properties of different soil types in study area; 2 ) to study how plant species and soil type affects the microbial diversity and abundance; 3 ) to explain the differences among tested habitats.

\section{Materials and Methods}

\section{Study site description and soil sampling:}

Soils were collected from three different sub-regions (Kunger, Gary and Wa- 
diAb-Gadad sub-regions in the Khartoum State, in arid/semi-arid parts in Central Sudan. The research work in this study had been carried out in arid/semiarid regions of Central Sudan, around latitude $15^{\circ} 50^{\prime} \mathrm{N}$ and longitude $32^{\circ} 55^{\prime} \mathrm{E}$. Soil samples were collected from $0-5 \mathrm{~cm}$ and $5-15 \mathrm{~cm}$ depths and kept in plastic bag. After collection, soil samples were brought to the laboratory and separated into two sub samples; one for bacteriological analysis that was kept in a refrigerator and the other one for the analysis of soil physico-chemical properties. Soil sampling was done in December, 2011.

\section{Bacteriological analysis:}

Nutrient agar medium was used for the enumeration of bacteria present in soil samples [7]. The $\mathrm{pH}$ was adjusted before addition of agar and sterilization. Serial dilution plate technique was used for the isolation of microorganism. One gram soilsample was diluted (1:100) with $100 \mathrm{ml}$ distilled water in a sterile conical flask and shaken well. One $\mathrm{ml}$ of this suspension was transferred to $9 \mathrm{ml}$ of sterile water for tenfold (1:10) dilution and by following serial dilution further diluted up to $10^{5}$ times. Plating in duplicate plates was made for each diluted sample. One $\mathrm{ml}$ of each of the diluted sample was taken in a sterilized petri dish by pipette. Then, molten agar medium was poured and mixed thoroughly by rotating the petri dish, first in one direction and then in the opposite direction. After setting the medium, the plates were inverted and incubated at $37^{\circ} \mathrm{C}$ for 48 $\mathrm{h}$ in an incubator then, the plates having well discrete colonies were selected for counting. The selected plates were placed on a colony counter (Digital colony counter, DC-8OSK1000086, Kayagaki, Japan) to count the number of colonies.

Tests:

Motility test was determined according to Cruickshank et al., 1975 [8]. Catalase test Oxidation-Fermentation test $(\mathrm{O} / \mathrm{F})$, Oxidase test, Sugar fermentation test, Voges-Proskauer test, Nitrate reduction test, Indole production test, Urease test, Citrate utilization were determined according to Barrow and Feltham 1993 [9]. Casein hydrolysis was determined by method described by Williams and Cross, 1971 [10]. Starch hydrolysis was performed according to Collins et al., 1995 [11]. Total a viable count of bacteria was determined [12].

\section{Isolation of Streptomyces:}

Isolation of Streptomyces was performed by the soil dilution plate technique [13]. In this technique; $1 \mathrm{~g}$ of each soil sample was taken in $9 \mathrm{ml}$ of sterilized distilled water in pre-sterilized test tube. Serial aqueous dilutions $\left(10^{-2}-10^{-7}\right)$ were prepared by transferring $1 \mathrm{ml}$ of the soil suspension into $9 \mathrm{ml}$ of sterilized distilled water in sterilized test tubes. Different aqueous dilutions $\left(10^{-4}\right.$ thro- $\left.10^{-6}\right)$ of the soil suspensions were applied separately into sterilized Petri-dishes and 20 $\mathrm{ml}$ of Starch-Casein Agar salt medium, $\mathrm{SCKNO}_{3}$, was added, mixed thoroughly and the plates were incubated at $28^{\circ} \mathrm{C}$ for $7-14$ days. $\mathrm{SCKNO}_{3}$ medium was prepared by dissolving $10 \mathrm{~g}$ soluble starch, $2 \mathrm{~g}$ dipotassium hydrogen ortho-phosphate, $2 \mathrm{~g}$ potassium nitrate, $2 \mathrm{~g}$ sodium chloride, $4 \mathrm{~g}$ casein, $0.05 \mathrm{~g}$ hydrated magnesium sulphate, $0.1 \mathrm{~g}$ calcium carbonate; $0.01 \mathrm{~g}$ hydrated ferric sulphate, $15 \mathrm{~g}$ agar in one liter of distilled water. The medium was sterilized by au- 
toclaving at $121^{\circ} \mathrm{C}$ for 15 minutes. Colonies characteristic of Streptomycetaceae (rough, chalky, powdery and with earth odour) that appeared on the incubated plates were selected, repeatedly sub-cultured for purification and stored at $4^{\circ} \mathrm{C}$ onto slants of $\mathrm{SCKNO}_{3}$ medium until further examinations.

\section{Analysis of soil physico-chemical properties:}

The $\mathrm{pH}$ of the soil was measured in a soil water suspension (1:2, soil:water). The electrical conductivity (EC) analysis was measured in the saturated extract. $\mathrm{Na}+$ and $\mathrm{K}+$ were determined photometrically. The exchangeable cations $\left(\mathrm{Ca}^{++}\right.$ and $\mathrm{Mg}^{++}$) were determined by Atomic Absorption Spectrophotometer (AAS, Perkin-Elmer, 047-1705. Saturated percentage (SP) were also determined [14]. Organic carbon content of the soil was determined by Wakely and Black method (Cited by Moghimi et al., [15]). Total nitrogen (\%) was determined by Kjeldahl method following extraction from $2 \mathrm{~g}$ soil with conc. $\mathrm{H}_{2} \mathrm{SO}_{4}$. The particle size analysis was carried out by the Pipette method (Cited by Moghimi et al., 2013 [15]).

Once the percentage of sand, silt, and clay is measured, the soil may be assigned a textural class using the table of textural soil types (cited by Subrahmanyam and Sambamurty) [16].

\section{Bacterial diversity measures:}

1/Shannon-Weiner Biodiversity Index:

Species diversity

$$
(H)=-\left(P_{i}\right)\left(\log _{2} P_{i}\right)
$$

where: $P=$ The proportion of all individuals in the sample which belongs the species $i$.

2/Simpson Index:

$$
D=1-\sum_{i-1} S(P i)^{2}
$$

where: $D$ is the index number; $S=$ the total number of species; $P=$ the proportion of all individuals in the sample which belongs to species $i$.

(Cited by Subrahmanyam and Sambamurty [16])

\section{The soil characteristics in AL-Gaeli region:}

\section{1) Kunger sub-region:}

The soil of this sub-region is predominantly clay loam. The $\mathrm{pH}$ of soil samples ranged from 7.49 to 7.34 . The EC values varied from $2.75-0.86 \mathrm{mmohs} / \mathrm{cm}$. The total nitrogen was in range $0.91-0.035$. Organic carbon range between 0.36 and 0.64\%. C:N ratio range between 6:1 and 13:1. The SP ranged from 50.7\% - 28.4\%. Sodium contents ranges between 0.906 and $2.958 \mathrm{Meq} / \mathrm{L}$. As for $\mathrm{K}$ it varies between 0.142 and $0.379 \mathrm{Meq} / \mathrm{L}$. Calcium contents was found to vary between 4 $19 \mathrm{Meq} / \mathrm{L}$. Magnesium contents was found to vary between 2 and $10 \mathrm{Meq} / \mathrm{L}$. P contents ranged between 3.2888 and $3.3636 \mathrm{ppm}$.

\section{2) Gary sub-region:}

The soil of this sub-region is predominantly sandy clay loam. The $\mathrm{pH}$ of soil samples ranged from 7.24 to 7.78 . The EC values varied from $0.267-0.53$ $\mathrm{mmohs} / \mathrm{cm}$. The total nitrogen was in range $0.028-0.14$. Organic carbon range 
between $0.26 \%$ and $0.65 \%$. C:N ratio range between 5:1 and 13:1. The SP ranged from $22.1 \%-31.4 \%$. Sodium contents ranges between 0.513 and $1.77 \mathrm{Meq} / \mathrm{L}$. As for $\mathrm{K}$ it varies between 0.104 and $0.372 \mathrm{Meq} / \mathrm{L}$. Calcium contents was found to vary between 1.5 - 3.50 Meq/L. Magnesium contents was found to vary between 0.25 and $1.5 \mathrm{Meq} / \mathrm{L}$. P contents ranged between 0.0 and $1.587 \mathrm{ppm}$.

\section{3) WadiAb Gadad sub-region:}

The soil of this sub-region is predominantly sandy clay loam. The $\mathrm{pH}$ of soil samples ranged from 7.51 to 7.63 . The EC values varied from $0.242-0.38$ $\mathrm{mmohs} / \mathrm{cm}$. The total nitrogen was in range $0.021-0.042$. Organic carbon range between $0.19 \%$ and $0.40 \%$. C:N ratio range between $7: 1$ and 17:1. The SP ranged from $22.5 \%$ - 26.3\%. Sodium contents ranges between 0.936 and $1.721 \mathrm{Meq} / \mathrm{L}$. As for $\mathrm{K}$ it varies between 0.085 and $0.124 \mathrm{Meq} / \mathrm{L}$. Calcium contents was found to vary between 1.5 - 4.5 Meq/L. Magnesium contents was found to vary between 0.5 and $2.0 \mathrm{Meq} / \mathrm{L}$. P contents ranged between 0.0 and $1.169 \mathrm{ppm}$.

\section{Results and Discussion}

The results concerning soil physical and chemical characteristics ( $\mathrm{pH}, \mathrm{EC}, \mathrm{SP}$, soluble cations: $\mathrm{Na}, \mathrm{K}, \mathrm{Ca}, \mathrm{Mg}$ and anion $\mathrm{P}$, organic carbon, total nitrogen and soil texture in three different studied sub-regions are presented in Tables 1-3.

Ten organisms were isolated from collected soil samples; Actinomyces spp., Streptomyces spp., Bacillus lentus, Bacillus badius, Bacillus pantothenticus, Bacillus circulans, Bacillus subtilis, Bacillus cereus, Micrococcus varians and

Table 1. Some soil physico-chemical properties of different samples from AL-Gaeli region-Kunger sub-region.

\begin{tabular}{|c|c|c|c|c|c|c|c|c|c|c|c|c|c|c|c|c|}
\hline $\begin{array}{l}\text { Sample } \\
\text { No. }\end{array}$ & $\begin{array}{c}\text { Soil } \\
\text { Depth }\end{array}$ & $\begin{array}{c}\text { Bacterial count } \\
(\mathrm{CFU} / \mathrm{g})\end{array}$ & $\mathrm{pH}$ & $\begin{array}{c}\text { EC } \\
\mathrm{mmohs} / \mathrm{cm}\end{array}$ & $\mathrm{N} \%$ & O.C \% & SP \% & $\begin{array}{c}\mathrm{Na} \\
\mathrm{Meq} / \mathrm{L}\end{array}$ & $\begin{array}{c}\mathrm{K} \\
\mathrm{Meq} / \mathrm{L}\end{array}$ & $\begin{array}{c}\mathrm{P} \\
\mathrm{ppm}\end{array}$ & $\begin{array}{c}\mathrm{Ca} \\
\mathrm{Meq} / \mathrm{L}\end{array}$ & $\mathrm{Mg}$ & $\begin{array}{c}\text { Clay } \\
\%\end{array}$ & $\begin{array}{l}\text { Silt } \\
\%\end{array}$ & Sand \% & $\begin{array}{c}\text { Textural Soil } \\
\text { Types }\end{array}$ \\
\hline KUN1 & $0-5$ & $8.5 \times 10^{5}$ & 7.35 & 2.75 & 0.077 & 0.46 & 28.4 & 2.958 & 0.577 & 2.455 & 28 & 11.5 & 33 & 24 & 43 & Clay loam \\
\hline KUN2 & $5-15$ & $3 \times 10^{4}$ & 7.35 & 1.82 & 0.042 & 0.4 & 50.7 & 2.898 & 0.385 & 0 & 17.5 & 3 & 33 & 22 & 45 & Clay loam \\
\hline KUN3 & $0-5$ & $8.5 \times 10^{5}$ & 7.41 & 0.86 & 0.049 & 0.63 & 28.4 & 0.906 & 0.295 & 2.125 & 6 & 2.5 & 29 & 16 & 56 & Sandy clay loam \\
\hline KUN4 & $5-15$ & $1.2 \times 10^{5}$ & 7.49 & 0.97 & 0.091 & 0.64 & 48.2 & 1.932 & 0.203 & 0 & 5.5 & 2.5 & 33 & 44 & 23 & Clay loam \\
\hline KUN5 & $0-5$ & $1.1 \times 10^{4}$ & 7.34 & 1.062 & 0.035 & 0.36 & 57.1 & 2.747 & 0.202 & 0 & 7 & 3.5 & 31 & 48 & 21 & Clay loam \\
\hline KUN6 & $5-15$ & $1.5 \times 10^{5}$ & 7.43 & 1.56 & 0.049 & 0.40 & 48.2 & 2.808 & 0.256 & 0 & 11.5 & 3.5 & 21 & 36 & 43 & Loam \\
\hline
\end{tabular}

Table 2. Some soil physico-chemical properties of different samples from AL-Gaeli region-Gary sub-region.

\begin{tabular}{|c|c|c|c|c|c|c|c|c|c|c|c|c|c|c|c|c|}
\hline $\begin{array}{l}\text { Sample } \\
\text { No. }\end{array}$ & $\begin{array}{c}\text { Soil } \\
\text { Depth }\end{array}$ & $\begin{array}{l}\text { Bacterial count } \\
(\mathrm{CFU} / \mathrm{g})\end{array}$ & $\mathrm{pH}$ & $\begin{array}{c}\text { EC } \\
\mathrm{mmohs} / \mathrm{cm}\end{array}$ & $\mathrm{N} \%$ & O.C $\%$ & ¿SP \% & $\begin{array}{c}\mathrm{Na} \\
\mathrm{Meq} / \mathrm{L}\end{array}$ & $\begin{array}{c}\mathrm{K} \\
\mathrm{Meq} / \mathrm{L}\end{array}$ & $\begin{array}{c}\mathrm{P} \\
\mathrm{ppm}\end{array}$ & $\begin{array}{c}\mathrm{Ca} \\
\mathrm{Meq} / \mathrm{L}\end{array}$ & $\begin{array}{c}\mathrm{Mg} \\
\mathrm{Meq} / \mathrm{L}\end{array}$ & $\begin{array}{c}\text { Clay } \\
\%\end{array}$ & $\begin{array}{l}\text { Silt } \\
\%\end{array}$ & Sand \% & $\begin{array}{c}\text { Textural Soil } \\
\text { Types }\end{array}$ \\
\hline GAR1 & $0-5$ & $1.7 \times 10^{2}$ & 7.45 & 0.32 & 0.14 & 0.65 & 25.5 & 0.513 & 0.181 & 1.587 & 3 & 1 & 20 & 2 & 78 & Sandy loam \\
\hline GAR2 & $5-15$ & $7.5 \times 10^{4}$ & 7.61 & 0.244 & 0.063 & 0.63 & 22.1 & 1.147 & 0.106 & 0.896 & 1.5 & 1 & 20 & 2 & 78 & Sandy loam \\
\hline GAR3 & $0-5$ & $1.5 \times 10^{4}$ & 7.24 & 0.267 & 0.042 & 0.46 & 31.4 & 0.845 & 0.154 & 1.10 & 2.5 & 1 & 24 & 22 & 54 & $\begin{array}{c}\text { Sandy clay } \\
\text { loam }\end{array}$ \\
\hline GAR4 & $5-15$ & $7.5 \times 10^{3}$ & 7.28 & 0.28 & 0.043 & 0.53 & 25.9 & 1.177 & 0.104 & 0 & 1.5 & 1 & 33 & 14 & 53 & $\begin{array}{l}\text { Sandy clay } \\
\text { loam }\end{array}$ \\
\hline GAR5 & $0-5$ & $1.2 \times 10^{5}$ & 7.71 & 0.53 & 0.049 & 0.64 & 26.3 & 1.087 & 0.372 & 0 & 3.5 & 1.5 & 20 & 9 & 71 & $\begin{array}{l}\text { Sandy clay } \\
\text { loam }\end{array}$ \\
\hline GAR6 & $5-15$ & $1.6 \times 10^{4}$ & 7.78 & 0.281 & 0.028 & 0.26 & 23.4 & 0.905 & 0.172 & 0 & 2 & 0.25 & 35 & 27 & 39 & Clay loam \\
\hline
\end{tabular}


Table 3. Some soil physico-chemical properties of different samples from AL-Gaeli region-WadiAb-Gadad sub-region.

\begin{tabular}{|c|c|c|c|c|c|c|c|c|c|c|c|c|c|c|c|c|}
\hline $\begin{array}{l}\text { Sample } \\
\text { No. }\end{array}$ & $\begin{array}{c}\text { Soil } \\
\text { Depth }\end{array}$ & $\begin{array}{l}\text { Bacterial count } \\
(\mathrm{CFU} / \mathrm{g})\end{array}$ & $\mathrm{pH}$ & $\begin{array}{c}\mathrm{EC} \\
\mathrm{mmohs} / \mathrm{cm}\end{array}$ & $\mathrm{N} \%$ & O.C $\%$ & $\% \begin{array}{l}S P \\
\%\end{array}$ & $\begin{array}{c}\mathrm{Na} \\
\mathrm{Meq} / \mathrm{L}\end{array}$ & $\begin{array}{c}\mathrm{K} \\
\mathrm{Meq} / \mathrm{L}\end{array}$ & $\begin{array}{c}\mathrm{P} \\
\mathrm{ppm}\end{array}$ & $\begin{array}{c}\mathrm{Ca} \\
\mathrm{Meq} / \mathrm{L}\end{array}$ & $\begin{array}{c}\mathrm{Mg} \\
\mathrm{Meq} / \mathrm{L}\end{array}$ & $\begin{array}{c}\text { Clay } \\
\%\end{array}$ & Silt & and $\%$ & $\begin{array}{c}\text { Textural Soil } \\
\text { Types }\end{array}$ \\
\hline ABG1 & $0-5$ & $6 \times 10^{4}$ & 7.61 & 0.34 & 0.038 & 0.28 & 26.3 & 1.721 & 0.117 & 0 & 2.5 & 0.5 & 19 & 23 & 59 & Sandy loam \\
\hline ABG2 & $5-15$ & $1 \times 10^{4}$ & 7.51 & 0.242 & 0.042 & 0.40 & 23.8 & 0.966 & 0.106 & 0 & 1.5 & 0.5 & 11 & 6 & 83 & Loamy Sand \\
\hline ABG3 & $0-5$ & $6.5 \times 10^{5}$ & 7.62 & 0.35 & 0.021 & 0.35 & 25.1 & 1.389 & 0.085 & 0.761 & 4.5 & 2 & 26 & 4 & 70 & Sandy clay loam \\
\hline ABG4 & $5-15$ & $5 \times 10^{3}$ & 7.63 & 0.38 & 0.028 & 0.19 & 22.5 & 0.936 & 0.124 & 1.169 & 3.5 & 2 & 20 & 1 & 80 & Sandy clay loam \\
\hline
\end{tabular}

Micrococcus roseus. Actinomycetes spp. have highest frequency in the three studied sub-regions and next are Streptomyces spp.

Total bacterial count of different soil samples from AL-Gaeli region are presented in Table 4. The diversity of soil microorganisms of the study habitat is presented in Table 5. The correlation effects between the soils parameters on bacterial count were studied (Table 6 and Table 7).

The Relationship between the soil physico-chemical properties and the viable bacterial count ( $\mathrm{cfu} \cdot \mathrm{g}^{-1}$ soil) in AL-Gaeli region

The correlation effects between the soil parameters on bacterial count were studied.

\section{1) Kunger sub-region:}

Total bacterial count was positively correlated $(\mathrm{p}=0.05)$ with $\mathrm{P}(\mathrm{r}=0.9876)$, sand $(r=0.6254), K(r=0.607), \mathrm{Ca}(r=0.6133), \mathrm{Mg}(\mathrm{r}=0.5644), \mathrm{OC}(\mathrm{r}=$ $0.4471)$, EC $(r=0.321)$ clay $(r=0.0637)$ and $N(r=0.0274)$ and negatively correlated $(\mathrm{p}=0.05)$ with $\mathrm{pH}(\mathrm{r}=-0.0907)$, silt $(\mathrm{r}=-0.6772)$ and $\mathrm{SP}(\mathrm{r}=-0.9844)$. Table 6.

\section{2) Gary sub-region:}

Total bacterial count was positively correlated $(\mathrm{p}=0.05)$ with $\mathrm{pH}(\mathrm{r}=0.5513)$, sand $(r=0.4464), K(r=0.6784)$, EC $(r=0.70), \mathrm{Na}(r=0.5269)$, Ca (0.03506), $\mathrm{Mg}(\mathrm{r}=0.06022)$ and $\mathrm{OC}(\mathrm{r}=0.4349)$ and negatively correlated $(\mathrm{p}=0.05)$ with clay, silt, SP, P and N. Table 7.

\section{3) WadiAb-Gadad sub-region:}

The sample size is too small to allow a reliable calculation of the Pearson Correlation Coefficient.

All the relationships between the total viable bacterial counts and soil physico-chemical properties or plant species are compiled in Table 6 and Table 7. There were obvious differences in correlation coefficients among the selected criteria (65\% from the total number of correlation coefficients were positively correlated between bacterial counts and soil physico-chemical properties.

Our results showed that microbial population was different in soil under different plant covers, soil types and depths. The total number of isolated bacteria varied in different samples of studied soils.

The higher bacterial counts CFUs observed in the Calotropis procera and Acacia tortilis ssp. rhizospheres $\left(8.5 \times 10^{5}\right)$ at surface layer of soil in Kunger sub-region and in Panicum turgidum rhizosphere $\left(7.5 \times 10^{4}\right)$ at sub-soil of Gary 
Table 4. Total bacterial count of different soil samples from AL-Gaeli region.

\begin{tabular}{|c|c|c|c|c|}
\hline Sample No. & Plant & Soil Depth & Bacterial count (CFU/g) & Type of bacteria isolated \\
\hline KUN1 & Capparis decidua & $0-5$ & $8.5 \times 10^{5}$ & $\begin{array}{l}\text { Bacillus circulans } \\
\text { Bacillus cereus } \\
\text { Actinomyces spp. } \\
\text { Streptomyces spp. }\end{array}$ \\
\hline KUN2 & Capparis decidua & $5-15$ & $3 \times 10^{4}$ & $\begin{array}{c}\text { B. lentus } \\
\text { B. circulans } \\
\text { B. cereus } \\
\text { Actinomyces spp. } \\
\text { Streptomyces spp. }\end{array}$ \\
\hline KUN3 & Acacia tortulis ssp. Samur & $0-5$ & $8.5 \times 10^{5}$ & $\begin{array}{c}\text { B. lentus } \\
\text { B. circulans } \\
\text { B. cereus } \\
\text { Actinomyces spp. } \\
\text { Streptomyces spp. }\end{array}$ \\
\hline KUN4 & Acacia tortulis ssp. Samur & $5-15$ & $1.2 \times 10^{5}$ & $\begin{array}{c}\text { B. lentus } \\
\text { B. circulans } \\
\text { B. subtilis } \\
\text { B. cereus } \\
\text { Actinomyces spp. } \\
\text { Streptomyces spp. }\end{array}$ \\
\hline KUN5 & Calotropis procera & $0-5$ & $1.1 \times 10^{4}$ & $\begin{array}{c}\text { B. circulans } \\
\text { B. subtilis } \\
\text { B. cereus } \\
\text { Actinomyces spp. } \\
\text { Streptomyces spp. }\end{array}$ \\
\hline KUN6 & Calotropis procera & $5-15$ & $1.5 \times 10^{5}$ & $\begin{array}{c}\text { B. lentus } \\
\text { B. subtilis } \\
\text { Micrococcus roseus } \\
\text { Actinomyces spp. } \\
\text { Streptomyces spp. }\end{array}$ \\
\hline GAR1 & Panicum turgidum & $0-5$ & $1.7 \times 10^{2}$ & $\begin{array}{c}\text { B. lentus } \\
\text { B. cereus } \\
\text { Micrococcus roseus } \\
\text { Actinomyces spp. } \\
\text { Streptomyces spp. }\end{array}$ \\
\hline GAR2 & Panicumturgidum & $5-15$ & $7.5 \times 10^{4}$ & $\begin{array}{c}\text { B. lentus } \\
\text { Micrococcus varians } \\
\text { Actinomyces spp. } \\
\text { Streptomyces spp. }\end{array}$ \\
\hline GAR3 & Acacia ehrenbergiana & $0-5$ & $1.5 \times 10^{4}$ & $\begin{array}{c}\text { B. cereus } \\
\text { B. badius } \\
\text { B. pantothenticus } \\
\text { Actinomyces spp. } \\
\text { Streptomyces spp. }\end{array}$ \\
\hline GAR4 & A. ehrenbergiana & $5-15$ & $7.5 \times 10^{3}$ & $\begin{array}{c}\text { B. cereus } \\
\text { Actinomyces spp. } \\
\text { Streptomyces spp. }\end{array}$ \\
\hline GAR5 & Leptadenia pyrotechnica & $0-5$ & $1.2 \times 10^{5}$ & $\begin{array}{c}\text { B. lentus } \\
\text { B. cereus } \\
\text { Actinomyces spp. } \\
\text { Streptomyces spp. }\end{array}$ \\
\hline
\end{tabular}




\section{Continued}

\begin{tabular}{|c|c|c|c|c|}
\hline GAR6 & Leptadenia pyrotechnica & $5-15$ & $1.6 \times 10^{4}$ & $\begin{array}{c}\text { B. badius } \\
\text { Actinomyces spp. } \\
\text { Streptomyces spp. }\end{array}$ \\
\hline ABG1 & Balanites aegyptiaca & $0-5$ & $6 \times 10^{4}$ & $\begin{array}{c}\text { B. lentus } \\
\text { B. circulans } \\
\text { Actinomyces spp. } \\
\text { Streptomyces spp. }\end{array}$ \\
\hline ABG2 & Balanites aegyptiaca & $5-15$ & $1 \times 10^{4}$ & $\begin{array}{c}\text { B. badius } \\
\text { Actinomyces spp. } \\
\text { Streptomyces spp. }\end{array}$ \\
\hline ABG3 & Acacia tortilis ssp. Radiana & $0-5$ & $6.5 \times 10^{5}$ & $\begin{array}{c}\text { B. lentus } \\
\text { B. subtilis } \\
\text { Actinomyces spp. } \\
\text { Actinomyces spp. } \\
\text { Streptomyces spp. }\end{array}$ \\
\hline ABG4 & Acacia tortilis ssp. Radiana & $5-15$ & $5 \times 10^{3}$ & $\begin{array}{c}\text { B. cereus } \\
\text { B. lentus } \\
\text { Actinomyces spp. } \\
\text { Streptomyces spp. }\end{array}$ \\
\hline
\end{tabular}

Table 5. Diversity of microorganisms in the study area.

\begin{tabular}{ccc}
\hline Sub-region & Shannon-Weiner Diversity Index & Simpson Diversity Index \\
\hline Kunger & 1.328 & 2.322 \\
Gary & 1.306 & 2.2115 \\
WadiAb Gadad & 0.4580 & 1.21 \\
\hline
\end{tabular}

Table 6. Correlation coefficients of the physico-chemical properties with the viable bacterial count (cfu $\cdot \mathrm{g}^{-1}$ soil) in Kunger sub-region.

\begin{tabular}{|c|c|c|c|}
\hline Soil Physico-chemical Properties & $\mathrm{R}$ & $\mathrm{R}^{2}$ & Correlation \\
\hline $\mathrm{pH}$ & -0.0907 & 0.0082 & $-\mathrm{ve}$ \\
\hline $\mathrm{EC}$ & 0.3212 & 0.1032 & Weak +ve \\
\hline Clay & 0.0637 & 0.0041 & Weak +ve \\
\hline Silt & -0.6772 & 0.4586 & $-\mathrm{ve}$ \\
\hline Sand & 0.6254 & 0.3911 & Moderate +ve \\
\hline SP & -0.9844 & 0.969 & $-\mathrm{ve}$ \\
\hline $\mathrm{Na}$ & -0.4419 & 0.1953 & - ve \\
\hline $\mathrm{K}$ & 0.607 & 0.3684 & Moderate +ve \\
\hline $\mathrm{P}$ & 0.9876 & 0.9754 & Strong +ve \\
\hline $\mathrm{Ca}$ & 0.6133 & 0.3761 & Moderate +ve \\
\hline $\mathrm{Mg}$ & 0.5644 & 0.3185 & Moderate +ve \\
\hline $\mathrm{N}$ & 0.274 & 0.0751 & Weak +ve \\
\hline O.C & 0.4471 & 0.1999 & Weak +ve \\
\hline
\end{tabular}

Correlation: strong $+\mathrm{ve}=0.8-1.0 ;$ moderate $+\mathrm{ve}=0.3-0.7 ;$ weak $+\mathrm{ve}=$ less than $0.3 ;$ negative $=-\mathrm{ve}(\mathrm{SPSS})$.

sub-region. This could be to better availability of nutrients and environmental conditions which favored their growth. 
Table 7. Correlation coefficients of the physico-chemical properties with the viable bacterial count (cfu. $\mathrm{g}^{-1}$ soil) in Gary sub-region.

\begin{tabular}{cccc}
\hline Soil Physico-chemical Properties & $\mathrm{R}$ & $\mathrm{R}^{2}$ & Correlation \\
\hline $\mathrm{pH}$ & 0.5513 & 0.3039 & Moderate $+\mathrm{ve}$ \\
$\mathrm{EC}$ & 0.7 & 0.49 & Moderate $+\mathrm{ve}$ \\
Clay & -0.5319 & 0.2829 & Moderate $-\mathrm{ve}$ \\
Silt & -0.3414 & 0.1166 & $-\mathrm{ve}$ \\
Sand & 0.4464 & 0.1993 & $+\mathrm{ve}$ \\
$\mathrm{SP}$ & -0.2134 & 0.0455 & $-\mathrm{ve}$ \\
$\mathrm{Na}$ & 0.5269 & 0.2776 & Moderate $+\mathrm{ve}$ \\
$\mathrm{K}$ & 0.6784 & 0.4602 & Moderate $+\mathrm{ve}$ \\
$\mathrm{P}$ & -0.3356 & 0.1126 & Weak $-\mathrm{ve}$ \\
$\mathrm{Ca}$ & 0.3506 & 0.1229 & Weak $+\mathrm{ve}$ \\
$\mathrm{Mg}$ & 0.6022 & 0.3626 & Moderate $+\mathrm{ve}$ \\
$\mathrm{N}$ & -0.226 & 0.0511 & Weak $-\mathrm{ve}$ \\
$\mathrm{O} . \mathrm{C}$ & 0.4349 & 0.1891 & Weak +ve \\
\hline
\end{tabular}

Correlation: strong $+\mathrm{ve}=0.8-1.0 ;$ moderate $+\mathrm{ve}=0.3-0.7 ;$ weak $+\mathrm{ve}=$ less than $0.3 ;$ negative $=-\mathrm{ve}$ (SPSS).

In general, bacterial CFUs tend to decrease with increase in soil depth. Decrease in the bacterial CFUs with increasing soil depth in some cases could be related to the organic carbon content of the soil as nutrients are declining with the increase in soil depth. The higher bacterial CRUs at the surface layer might be due to the presence of litters, twigs, herbs and tree canopy which render a moist environment in the soil and favor high microbial activity and hence high microbial populations.

From the three sub-regions collected soils, five different textural soil classes (clay loam, sandy clay loam, loam, sandy loam and loamy sand) were detected. The data of soil $\mathrm{pH}$ values showed some differences among different soil textures. In study region, the lowest value $(\mathrm{pH}=7.24)$ was recorded in sandy clay loam and the highest one $(\mathrm{pH}=7.78)$ in clay loam. The highest value of soil organic carbon content were recorded in the texture soils (sandy loam, clay loam and sandy clay loam) whereas the lowest contents were in clay loam and sandy clay loam at sub-surface layer. These differences were documented previously by Silver et al., (2000) [17], who found that soil texture plays a key role in below ground $\mathrm{C}$ storage in soil ecosystems and strongly influences nutrient availability and retention, particularly in fine textural soils. Matus et al., (2008) [18] observed that soil organic carbon tends to be associated with the fine fraction of soils and it was significantly three times in clay-rich soils than coarse soils. Fine texture soil shows more stable aggregates, which in turn may act as a media of greater amount of organic carbon and total nitrogen contents (Raiesi, 2006) [19]. Based on the results, it appears that microbial biomass is influenced by soil texture.

\section{Conclusions}

Qualitative analysis of microorganisms isolated from the studied soil samples 
reveal a total of 8 different species of bacteria, of which 2 are unidentified. The 6 species are classified under Bacillus genera and the remaining 2 species are classified under Micrococcus genera.

The quantitative analysis of the isolated microorganisms was also carried out by considering individual colonies as separate units (CFUs). The quantitative data on microbial population recorded in the present study was analyzed using two diversity indices. High Shannon-Weiner diversity Index value for bacteria was obtained in Kunger sub-region (1.328) followed by Gary sub-region (1.306) and then WadiAb-Gadad sub-region (0.4580).

Soil Actinomyces spp. and Streptomyces spp. were the most abundant microorganisms identified in the three habitats (sub-regions) which directly may influence decomposition processes and nutrient cycling in the soil.

There are little variation in the occurrences of microorganisms in the studied sub-regions in terms of abundance and diversity. There is a need for greater understanding of physical, chemical, biochemical and biological factors influencing abundance and diversity on microbial habitats. Current biotechnology research is needed for developing new microbial pesticides from these studied microorganisms. The formulation of an appropriate national strategy in biotechnology should constitute an important, initial step towards the utilization and industrialization of microorganisms.

\section{References}

[1] Hassan, H.I. and EL-Kamali, H.H. (2015) Effect of Soil Physic-Chemical Properties and Plant Type on Bacterial Diversity in Semi-Arid Parts in Central Sudan. Part I. Omdurman North Region. Open Access Library Journal, 2, e1863. https://doi.org/10.4236/oalib.1101863

[2] Hassan, H.I. and EL-Kamali, H.H. (2016) Effect of Soil Physicochemical Properties and Plant Type on Bacterial Diversity in Semi-Arid Parts in Central Sudan. Part II. Sharq EL-Neel Region, Khartoum State. International Journal of Scientific World, 4, 4-10.

[3] Pettersson, M. (2004) Factors Affecting Rates of Change in Soil Bacterial Communities. Doctoral Thesis, Lund University, Lund.

[4] Bressan, M., Mougel, C., Dequiedt, S., et al. (2000) Response of Soil Bacterial Community Structure to Successive Perturbations of Different Types and Intensities. Environmental Microbiology, 10, 2184-2187. https://doi.org/10.1111/j.1462-2920.2008.01641.x

[5] Crecchio, C., Curci, M., Pellegrino, A., et al. (2007) Soil Microbial Dynamics and Genetic Diversity in Soil under Monoculture Wheat Grown in Different Long-Term Management Systems. Soil Biology and Biochemistry, 39, 1391-1400. https://doi.org/10.1016/j.soilbio.2006.12.016

[6] Hackl, E., Zeckmeister, E., Boltenstem, S., Bodrossy, L. and Sessitsch, A. (2004) Comparison of Diverties and Compositions of Bacterial Populations Inhabiting Natural Forest Soils. Applied and Environmental Microbiology, 70, 5057-5065. https://doi.org/10.1128/AEM.70.9.5057-5065.2004

[7] Cheesbrough, M. (1984) Culture Media. In: Cheesbrough, M., Ed., Medical Laboratory Manual for Tropical Countries, Vol. 3, Tropical Health Technology and Butterworth-Heineman, Cambridge, 60-69, 407-428. 
[8] Cruickshank, R. (1975) Medical Microbiology: A Guide to Diagnosis and Control of Infection. E and S Livingston Ltd., Edinburgh and London, 888.

[9] Barrow, G.H. and Feltham, R.K.A. (1993) Cowan and Steels Manual for Identification of Medical Bacteria. 3rd Edition, Cambridge University Press, Cambridge, 331.

[10] Williams, S.T. and Cross, T. (1971) Actinomycetes. In: Booth, C., Ed., Methods in Microbiology, Academic Press, London.

[11] Collins, C.H., lyne, P.M. and Grange, J. (1995) Collins and Laynes Microbiological Methods. Butterworth-Heinemann, London.

[12] Benson, T. (2001) Microbiological Applications Laboratory Manual in General Microbiology. 8th Edition, The McGraw-Hill, New York.

[13] You, K.M. and Park, Y.K. (2004) A New Method for the Selective Isolation of Actinomycetes from Soil. Biotechnology Techniques, 10, 541-546.

[14] Richards, L.A. (1954) Diagnosis and Improvement of Saline and Alkali Soils. Regional Salinity Laboratory, Washington DC.

[15] Moghimi, A.H., Hamdan, J., Shamshuddin, J., Samsuri, A.W. and Abtahi, A. (2013) Physico-Chemical Properties and Surface Charge Characteristics of Arid Soils in Southern Iran. Applied and Environmental Soil Science, 2013, Article ID: 252861. https://doi.org/10.1155/2013/252861

[16] Subrahmanyam, N.S. and Sambamurty, A.V.S.S. (2000) Ecology. Narosa Publishing House, New Delhi, 172.

[17] Silver, W., Neff, J., McGroddy, M., Veldkamp, E., Keller, M. and Cosme, R. (2000) Effects of Soil Texture on Belowground Carbon and Nutrient Storage in Lowland. Amazonian Forest Ecosystem. Ecosystems, 3, 193-209.

https://doi.org/10.1007/s100210000019

[18] Matus, F.J., Christopher, H.L. and Christian, R.M. (2008) Effect of Soil Texture, Carbon Input Rates, and Litter Quality on Free Organic Matter and Nitrogen Mineralization in Chilean Rain Forest and Agricultural Soils. Communications in Soil Science and Plant Analysis, 39, 187-201.

https://doi.org/10.1080/00103620701759137

[19] Raiesi, F. (2006) Carbon and N Mineralization as Affected by Soil Cultivation and Crop Residue in a Calcareous Wetland Ecosystem in Central Iran. Agriculture, Ecosystems \& Environment, 112, 13-20. https://doi.org/10.1016/j.agee.2005.07.002 
Submit or recommend next manuscript to OALib Journal and we will provide best service for you:

- Publication frequency: Monthly

- 9 subject areas of science, technology and medicine

- Fair and rigorous peer-review system

- Fast publication process

- Article promotion in various social networking sites (LinkedIn, Facebook, Twitter, etc.)

- Maximum dissemination of your research work

Submit Your Paper Online: Click Here to Submit

Or Contact service@oalib.com 